\title{
Rapid Assessment of Quality Changes in French Fries during Deep-frying Based on FTIR Spectroscopy Combined with Artificial Neural Network
}

\author{
Lirong $\mathrm{Xu}^{\#}$, Xue Mei", Jiarui Chang, Gangcheng Wu, Qingzhe Jin, and Xingguo Wang* \\ Collaborative Innovation Center of Food Safety and Quality Control in Jiangsu Province, National Engineering Research Center for Functional \\ Food, School of Food Science and Technology, Jiangnan University, 1800 Lihu Avenue Wuxi, Jiangsu 214122, P.R. CHINA
}

\begin{abstract}
Fourier transform infrared (FTIR) spectroscopy combined with backpropagation artificial neural network (BP-ANN) were utilized for rapid and simultaneous assessment of the lipid oxidation indices in French fries. The conventional indexes (i.e. total polar compounds, oxidized triacylglycerol polymerized products, oxidized triacylglycerol monomers, triacylglycerol hydrolysis products, and acid value), and FTIR absorbance intensity in French fries were determined during the deep-frying process, and the results showed the French fries had better quality in palm oil, followed by sunflower oil, rapeseed oil and soybean oil. The FTIR spectra of oil extracted from French fries were correlated to the reference oxidation indexes determined by AOCS standard methods. The results of BP-ANN prediction showed that the model based on FTIR fitted well $\left(R^{2}>0.926\right.$, RMSEC $\left.<0.481\right)$ compared with partial least-squares model $\left(R^{2}>0.876\right)$. This facile strategy with excellent performance has great potential for rapid characterization quality of French fries during frying.
\end{abstract}

Key words: French fries, polar compounds, oxidation indexes, BP-ANN, FTIR

\section{Introduction}

Deep frying is a traditional and convenient cooking method for producing palatable and desirable foods with unique characteristics of flavor, color and texture ${ }^{1)}$. Frying oils, exposed to high temperature $\left(150-200^{\circ} \mathrm{C}\right)$, are subject to complex reactions such as polymerization, oxidation, and hydrolysis ${ }^{2}$. These reactions produce polar compounds including oxidized triacylglycerol polymerized products (TGP), oxidized triacylglycerol monomers (OTG), and triacylglycerol hydrolysis products (THP $)^{3,4)}$. Therefore, those polar compounds were absorbed by the food during frying, which reduced the nutritional quality of the fried foods and might jeopardize human health ${ }^{5,6)}$. Numerous attentions have been paid on the oxidation degree of frying oils and ignored the lipid oxidation of fried food during deep-frying $^{7)}$. The fat content in fried food is exchanged with the used frying oil and the oxidation degree of frying oil affected the fried food by the fat absorption ${ }^{8}$. Therefore, more attention should be paid to the quality of fried food. Currently, the traditional determination of lipid oxidation degree during the deep-frying process is characterized samples by acid values (AV), total polar compounds (TPC) and the main fractions of polar compounds. However, these conventional methods are laborious and time-consuming with chemicals and fail to achieve the facile application in the frying industry ${ }^{9,10)}$.

For rapid and simultaneous determination of lipid oxidation in food, Fourier transform infrared (FTIR) spectroscopy can provide a solution for this challenge. FTIR spectroscopy was widely applied in the food detection due to environmental-friendliness, low-cost, and tiny sample and easy preparation for determination ${ }^{11-13)}$. FTIR has also been used to determine TPC content of frying oil ${ }^{14-16)}$. Artificial neural network (ANN) is an algorithm that models non-linear relationships by mimicking the functionality of a neural network, which contains 3 parts of the input layer, the hidden layer and the output layer and is typically specified by architecture, learning algorithm and neuron model. ANN has many advantages, e.g able to handle data with

Abbreviations: TPC, total polar compounds; TGP, oxidized triacylglycerol polymerized products; OTG, oxidized triacylglycerol monomers; THP, triacylglycerol hydrolysis products; AV, acid values; BP-ANN, back propagation artificial neural network

\footnotetext{
"Lirong Xu and Xue Mei are the co-first authors of the article.

* Correspondence to: Xingguo Wang, Collaborative Innovation Center of Food Safety and Quality Control in Jiangsu Province, National Engineering Research Center for Functional Food School of Food, Science and Technology,

Jiangnan University, 1800 Lihu Avenue Wuxi, Jiangsu 214122, P.R. CHINA

E-mail: wangxg1002@gmail.com; xingguow@jiangnan.edu.cn.

Accepted June 23, 2021 (received for review February 4, 2021)

Journal of Oleo Science ISSN 1345-8957 print / ISSN 1347-3352 online

http://www.jstage.jst.go.jp/browse/jos/ http://mc.manusriptcentral.com/jjocs

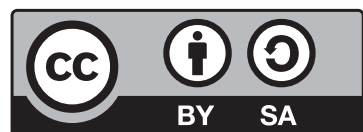


high collinearity and noise, able to model complex nonlinear relationships between independent and dependent variables, Training algorithm allows neural network to 'self-learn', able to detect interactions between predictor variables, requires less formal statistical training to develop ${ }^{17)}$. ANN has been applied successfully to the automation and intelligent control of food drying process ${ }^{18)}$, and food authentication ${ }^{19,20)}$, due to its self-learning ability, adaptive nature, strong fault tolerance ${ }^{21,22)}$. Karaman et al. estimated the oxidation parameters (peroxide value, free fatty acids and iodine value) of sunflower oil added with some natural byproduct extracts combined with $\mathrm{ANN}^{23)}$. However, to the best of our knowledge, there is limited research about FTIR spectroscopy combined with artificial neural network for the rapid and simultaneous determination of oxidation indexes in French fries.

In this study, FTIR spectroscopy in association with the back propagation artificial neural network (BP-ANN) model was developed for the routine analysis of TPC, TGP, THP, OTG, and AV of French fries fried in palm oil (F-PO), sunflower oil (F-SuO), soybean oil (F-SO) and rapeseed oil (F-RO). The feasibility and sensitivity of the method were evaluated and compared with the partial least-squares (PLS) model. The FTIR spectroscopy method will provide an alternative approach for monitoring the quality changes of French fries rapidly, contributing to the safety of fried food and scientific frying during deep-frying.

\section{Materials and Methods}

\subsection{Materials and reagents}

Fresh potatoes (Solanum tuberosum L, Helan15 cultivar), were obtained from a farm in Shandong Province, China. The frying experiments were conducted with soybean oil (SO), rapeseed oil (RO), palm oil(PO), and sunflower oil ( $\mathrm{SuO}$ ) (without additives) donated by Wilmar, and stored at $4^{\circ} \mathrm{C}$ for further use. Acetone, diethyl ether, petroleum ether, toluene, hexane (analytical reagent grade) and Tetrahydrofuran (chromatography grade) were purchased from Sinopharm Chemical Regent Co., Ltd (Shanghai, China) and Sigma (St. Louis, MO, USA), respectively.

\subsection{Preparation of French fries and oil extraction from French fries}

A stainless deep fryer (Aigoli, China) with a maximum capacity of $2.5 \mathrm{~L}$ was used for frying. Potatoes were washed and cut into pieces $(1 \times 1 \times(6 \pm 0.5) \mathrm{cm})$ with a manual cutter. Firstly, 2.0 L of fresh oil was heated to $168 \pm 3^{\circ} \mathrm{C}$ in the fryer. Then, a batch of $100 \pm 2 \mathrm{~g}$ of potato strips was fried in oils regularly. Frying time (4 min) and waiting time (26 min)were set in each frying batch. The overall time was 3 days $(8$ h/day). Frying oils (SO, RO, PO, and SuO) at different frying time of $0.06,2,4,6,8,10,12,14,16,18$, 20, 22 , and $24 \mathrm{~h}$ were used for French fries frying. Extraction of oil from the French fries was performed according to the method described by Li et al. ${ }^{8)}$. Those samples were stored at $-20^{\circ} \mathrm{C}$ until analysis.

\subsection{The physicochemical properties of French fries}

The fatty acid composition was analyzed by a gas chromatography (Agilent 7820, USA) (Table S1). The conditions were performed according to our previous research ${ }^{24)}$. $\mathrm{AV}$ of samples were measured according to the AOCS official method $3 \mathrm{a}-63^{25)}$. The determination of TPC was based on the AOCS official method Cd 20-9126). The compositions of TPC (e.g., TGP, OTG, THP) in oils extracted from French fries were obtained by a high-performance size-exclusion chromatography method described by our previous study ${ }^{27)}$.

\subsection{FTIR analyses}

A Bruker FTIR spectrometer(VERTEX 70 series) was employed to obtain the FTIR spectra. Each recorded spectrum was obtained by averaging 16 scans at a resolution of $4 \mathrm{~cm}^{-1}$. Each sample was measured three times. The average of the three spectra obtained from each same sample was used in subsequent analysis. The spectra file of samples was pretreated through normalization to reduce measurement error. Each spectrum was aligned to the baseline using instrumentation software. $100 \mu \mathrm{L}$ of oil extracted from the French fries was deposited onto PE film surface to form a uniform film. With PE film as background, the spectrum of oil was obtained. Using the calibration equation associating the effective path length with the absorbance of $4334 \mathrm{~cm}^{-1}$, the effective path lengths of spectra were normalized to $0.15 \mathrm{~mm}^{28)}$.

\subsection{Artificial neural network modeling and partial least- squares model}

The BP-ANN is a multi-layer feedforward neural network trained based on the error back propagation algorithm ${ }^{29}$. It is composed of an input layer, hidden layer, and output layer. In general, the three-layer network can approach the target value with arbitrary precision if the number of hidden layers is sufficient. It is typically specified by architecture, learning algorithm and neuron model, in which architecture represents the interconnection pattern between the different layers of neurons, learning algorithm is for updating the weights in order to correctly model a particular task and neuron model defined by activation function is to transform a neuron's weighted input to its output activation. Through parameter optimization in the pre-experiment, the best BP-ANN model was obtained with the neuron number of 10 in a hidden layer, the training function of trainlm, and the transfer functions of tansig and purelin in hidden layer and output layer, respectively. 
Therefore, a three-layer network was selected for all neural network models in this study, and 156 sets of data were obtained and divided into training data (70\%, 110 samples), and validation data (15\%, 23 samples), and testing data (15\%, 23 samples). Based on our preliminary experiment and previous study ${ }^{30)}$, the FTIR absorbance peak at 1696 $\mathrm{cm}^{-1}, 3471 \mathrm{~cm}^{-1}$ and $968 \mathrm{~cm}^{-1}$ related to oil oxidation were used as inputs of BP-ANN model to predict the conventional indexes (output) (TPC, TGP, THP, OTG, and AV). The partial least-squares (PLS) model was also validated using the FTIR absorbance peak height at $1696 \mathrm{~cm}^{-1}, 3471$ $\mathrm{cm}^{-1}$ and $968 \mathrm{~cm}^{-1}$ and these relevant conventional indexes. Root Mean square error (RMSE), coefficient of determination $\left(\mathrm{R}^{2}\right)$ of the predicted conventional indexes were computed to evaluate the performance of fitting and predicting. Furthermore, in order to compare the quality of BP-ANN model and PLS, root mean square error of calibration (RMSEC) and root mean square error of prediction (RMREP) were calculated for the evaluation. Besides 10 blind French fries samples were collected as external validation samples.

\subsection{Statistical analyses}

The reference values and the spectra collected of the samples were conducted in triplicate. The relevant results were denoted as mean \pm standard deviation (SD). BP-ANN was performed using MATLAB R2014a(The MathWorks
Inc., Natick, MA, USA). The PLS model was performed using SIMCA-P (version 13.0, Umea, Sweden). Spectral data analyses were conducted with the use of OMNIC 7.3 (Thermo Electron Inc., Madison, WI).

\section{Results and Discussion}

\subsection{Results of chemical analysis}

Before FTIR analysis, the TPC, TGP, THP, OTG, and AV of oil samples from French fries were determined. Here, the degree of thermal oxidative decomposition in French fries fried in frying oils at $0.06,4,8,12,16,20 \mathrm{~h}$ are displayed in Fig. 1.

With a longer period of the frying process, the TPC in French fries kept increasing but the increase rates declined gradually in general. For TPC, the content of polar compounds in F-SO was consistently higher than that in $\mathrm{F}-\mathrm{RO}$, $\mathrm{F}-\mathrm{SuO}$, and F-PO, the polar compounds in F-SuO and F-PO was the lowest during frying. The F-SO reached $26.64 \%$ at 20 h frying compared with F-PO with TPC of $19.61 \%$. (Fig. 1A). The TGP contents in F-SO, F-RO, F-SuO and F-PO reached to $13.24 \%, 10.59 \%, 10.14 \%$ and $9.83 \%$ in the three days of frying, and the order of growth rate was F-SO $(0.43 \%)>\mathrm{F}-\mathrm{RO}(0.34 \%)>\mathrm{F}-\mathrm{SuO}(0.32 \%)>\mathrm{F}-\mathrm{PO}(0.31$ $\%$ ) (Fig. 1B). The OTG contents in F-SO and F-RO increased rapidly at the first $8 \mathrm{~h}$ and then increased slowly.
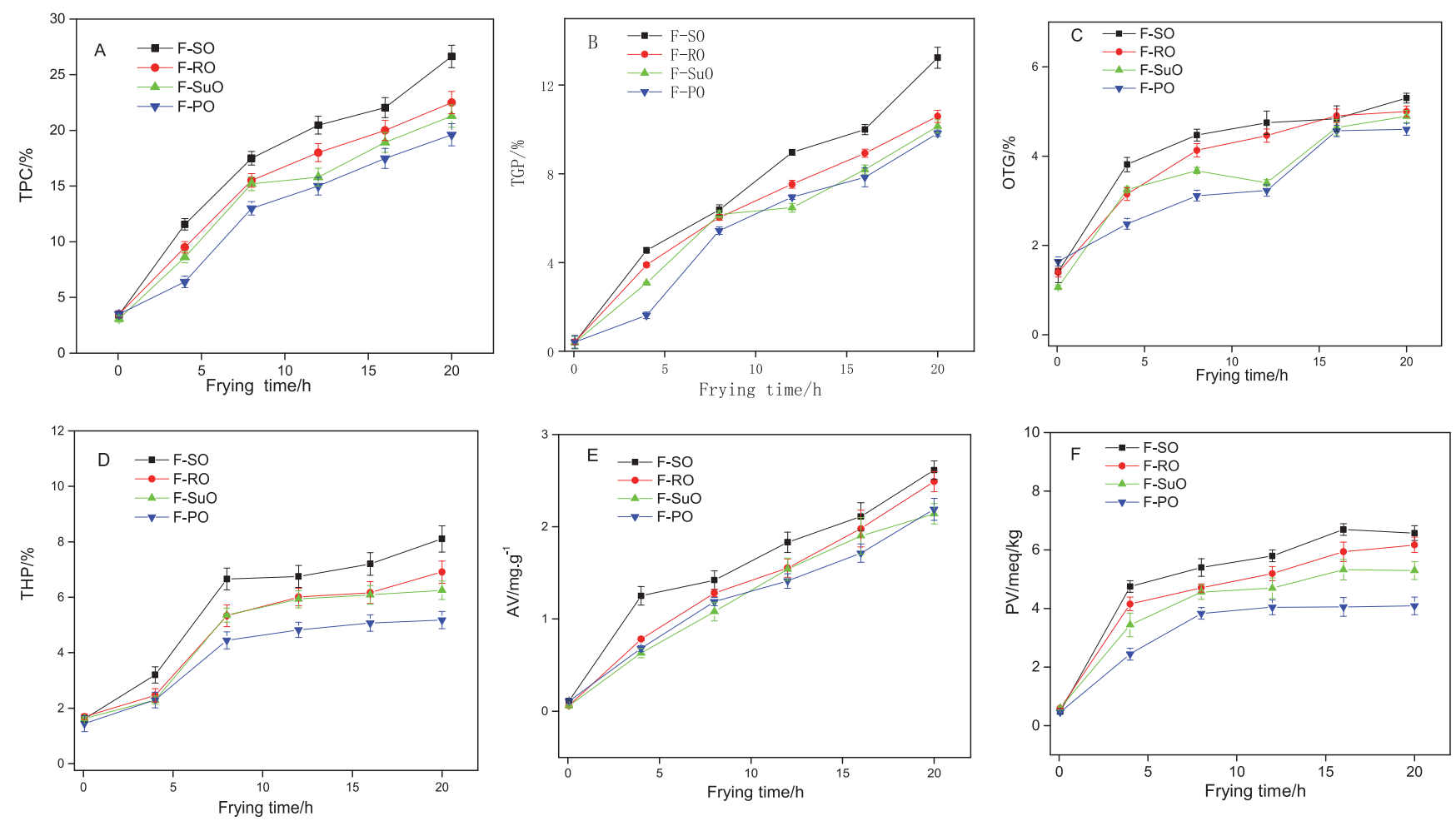

Fig. 1 TPC (A), TGP (B), OTG (C), THP (D), AV (E) and PV (F) of French fries fried in soybean oil (F-SO), rapeseed oil (F-RO), sunflower oil (F-SuO), palm oil (F-PO) at different frying periods. 
While, the OTG contents greatly fluctuated in F-SuO and F-PO and their contents were lower than F-SO and F-RO at the primary frying period (before $15 \mathrm{~h}$ ) (Fig. 1C). The THP contents in French fries increased exponentially with the frying time. The order of THP average growth rates were F-SO $(0.22 \%)>\mathrm{F}-\mathrm{RO}(0.17 \%)>\mathrm{F}-\mathrm{SuO}(0.15 \%)>$ F-PO (0.12\%) (Fig. 1D). The AV increased with the frying time as presented in Fig. 1E, and F-SO presented a higher value compared to other samples.

\subsection{FTIR analysis}

FTIR spectroscopy is a rapid method for food detection, which is an alternative to traditional methods in the food industries. The complete FTIR spectra of oil samples extracted from French fries were exhibited in Fig. 2A. For lipid oxidation analysis, the most essential spectral regions correspond to $\mathrm{ROOH}, \mathrm{FFA}(\mathrm{C}=\mathrm{O}$ bond $)$, and $-\mathrm{HC}=\mathrm{CH}-$ (trans) functional groups ${ }^{31)}$. More specifically, the corresponding regions were the following wavenumbers: the $-\mathrm{HC}=\mathrm{CH}-$ (trans) bond occurred at nearly $980-960 \mathrm{~cm}^{-1}$; the FFA $(\mathrm{C}=\mathrm{O}$ bond $)$ occurred between $1720-1680 \mathrm{~cm}^{-1}$ and the ROOH corresponded to the regions of 3650-3300 $\mathrm{cm}^{-131-33)}$. The change in intensity of these spectral regions of oil samples could reflect the chemicals transformation during frying, consequently denoting the degree of lipid oxidation.

Functional group spectra's variation for samples in the course of frying was shown in Fig. 2A. The FFA $(\mathrm{C}=\mathrm{O}$ bond) absorbance at about $1696 \mathrm{~cm}^{-1},-\mathrm{HC}=\mathrm{CH}$-(trans) bond absorbance at about $968 \mathrm{~cm}^{-1}$ and ROOH characteristic absorption peak height at about $3471 \mathrm{~cm}^{-1}$ of oils extracted from French fries showed the growth trend during frying. The starting adjacent spectral gaps at the height of these absorption peaks were small, whereas the adjacent spectral gaps increased with time, suggesting that hydrogen peroxide, free fatty acids and isolated trans fatty acids increased with time (Figs. S1 (A-C)). Figures 2B-2D showed that F-PO and F-SuO displayed the lower characteristic absorption peak height at 3471, 1696 and $968 \mathrm{~cm}^{-1}$ compared with F-SO and F-RO by comparing the FTIR ab-
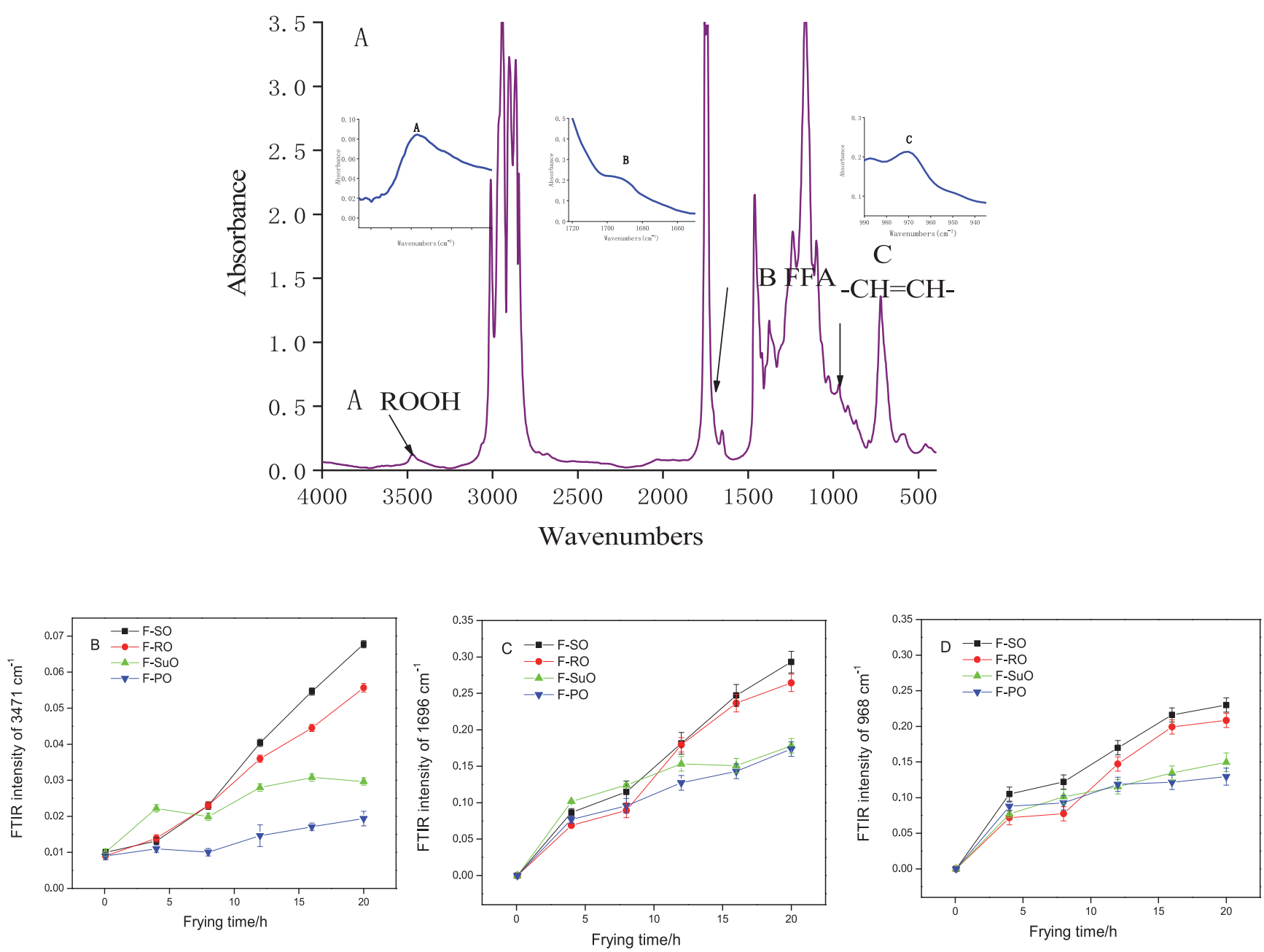

Fig. 2 Change of the functional group spectra for French fries sample fried in $\mathrm{SO}(\mathrm{A})$, changes of absorbance peak at $3471 \mathrm{~cm}^{-1}(\mathrm{~B}), 1696 \mathrm{~cm}^{-1}(\mathrm{C}), 968 \mathrm{~cm}^{-1}$ (D) for French fries fried in different oils during frying. 
sorbance intensity of the $\mathrm{ROOH}, \mathrm{FFA}(\mathrm{C}=\mathrm{O}$ bond $)$, and $-\mathrm{HC}=\mathrm{CH}-$ (trans) bond in French fries samples, the results revealed that the degree of oxidative stability was F-PO> $\mathrm{F}-\mathrm{SuO}>\mathrm{F}-\mathrm{RO}>\mathrm{F}-\mathrm{SO}$.

\subsection{Model establishment and verification by BP-ANN}

To establish the BP-ANN model for the change of quality in French fries during deep-frying, the FTIR absorbance peak at 1696, 3471 and $968 \mathrm{~cm}^{-1}$, were set as variable X. Meanwhile, the conventional indexes (TPC, TGP, THP, OTG, AV) were set as variables Y. Through parameter optimization, the BP-ANN model with the topology of 3-10-1 (the neuron number of the input layer, hidden layer, and output layer was 3, 10, and 1, respectively), performing algorithm of Levenberg-Marquardt, the transfer function of tansig and purelin in hidden layer and output layer, respec- tively, and training function of trainlm (Levenberg-Marquardt) outperformed the fitting performance and accuracy. Training set included $70 \%$ of the samples, and the prediction models of TPC, TGP, THP, OTG, and AV established by BP-ANN method are shown in Fig. 3. The predicted values were well correlated with the actual values, and the $\mathrm{R}^{2}$ of the training set were all $>0.915 .15 \%$ of the samples were used as the validation set and $15 \%$ as a test set. The results showed that the $\mathrm{R}^{2}$ of the validation set and testing set of TPC, TGP, THP, AV were $>0.945$ and $>$ 0.940, respectively, indicating that the prediction ability of the model was available (Table 1), numerous studies revealed that the $\mathrm{R}^{2}$ higher than 0.9 in the model indicated excellent classification performance as well as prediction ability ${ }^{34)}$. However, $\mathrm{R}^{2}$ of the testing set of OTG was only 0.863 , which is not good owing to the fluctuation of OTG
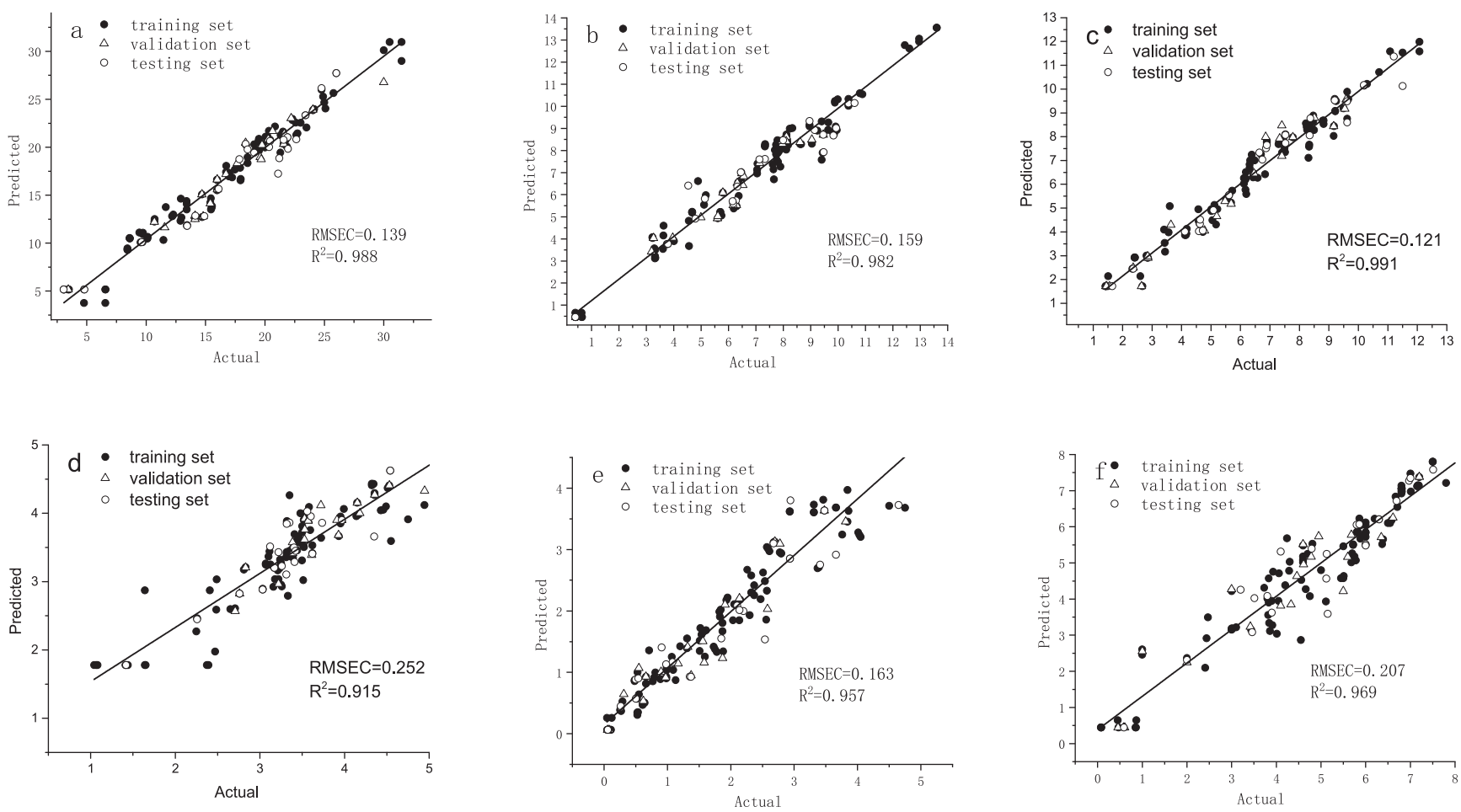

Fig. 3 Establishment of prediction model by BP-ANN. (a) The prediction model of TPC; (b) The prediction model of TGP; (c) The prediction model of THP; (d) The prediction model of AV; (e) The prediction model of PV.

Table 1 Performance of BP-ANN model.

\begin{tabular}{|c|c|c|c|c|c|c|}
\hline \multirow{2}{*}{ Index } & \multicolumn{2}{|c|}{ Training } & \multicolumn{2}{|c|}{ Validation } & \multicolumn{2}{|c|}{ Testing } \\
\hline & MSE & $\mathrm{R}^{2}$ & MSE & $\mathrm{R}^{2}$ & MSE & $\mathrm{R}^{2}$ \\
\hline $\mathrm{TPC}$ & 0.139 & 0.988 & 0.207 & 0.977 & 0.203 & 0.965 \\
\hline TGP & 0.159 & 0.982 & 0.209 & 0.977 & 0.206 & 0.968 \\
\hline THP & 0.121 & 0.991 & 0.149 & 0.985 & 0.159 & 0.972 \\
\hline OTG & 0.252 & 0.915 & 0.477 & 0.895 & 0.481 & 0.863 \\
\hline AV & 0.163 & 0.957 & 0.287 & 0.945 & 0.242 & 0.940 \\
\hline
\end{tabular}

MSE: Mean Square Error, $\mathrm{R}^{2}$ : coefficient of determination 
Table 2 Performance comparison of PLS and BP-ANN models.

\begin{tabular}{ccccc}
\hline Index & Model & $\mathrm{R}^{2}$ & RMSEC & RMSEP \\
\hline \multirow{2}{*}{ TPC } & PLS & 0.910 & 0.644 & 0.657 \\
& BP-ANN & 0.977 & 0.214 & 0.250 \\
TGP & PLS & 0.913 & 0.732 & 0.765 \\
& BP-ANN & 0.979 & 0.344 & 0.310 \\
THP & PLS & 0.896 & 0.577 & 0.513 \\
& BP-ANN & 0.987 & 0.184 & 0.124 \\
OTG & PLS & 0.876 & 0.889 & 0.833 \\
& BP-ANN & 0.926 & 0.481 & 0.464 \\
AV & PLS & 0.917 & 0.422 & 0.418 \\
& BP-ANN & 0.955 & 0.134 & 0.130 \\
\hline
\end{tabular}

$\mathrm{R}^{2}$ : coefficient of determination; RMSEC: root mean square error of calibration; RMSEP: root mean square error of prediction

with frying time. From Table 1, the MSE of the TPC, TGP, THP, and AV training models were 0.139, 0.159, 0.121, and 0.163 , respectively, and the MSE of validation and testing models were $<0.287$ and $<0.242$, respectively, which indicated that BP-ANN models had an excellent predictive ability for oxidation indexes in French fries during deepfrying. Furthermore, to evaluate the performance of the model, the results of BP-ANN models were compared with
PLS models (Table 2). Compared with PLS model, the RMSEC and RMSEP of the BP-ANN model were reduced by $45.9 \%-68.2 \%$ and $44.3 \%-68.9 \%$, and the $\mathrm{R}^{2}$ value was increased by $4.14 \%-10.15 \%$, respectively. In our study, the lower RMSEC, RMSEP and higher $\mathrm{R}^{2}$ of the calibration (training) equations for TPC, TGP, THP, OTG, AV were obtained compared with PLS models. The BP-ANN had achieved excellent predictive performance $\left(\mathrm{R}^{2}>0.926\right)$ for the TPC, TGP, THP, AV conventional indexes during the frying process.

\subsection{Sample external validation}

After calibrating the models, external validation procedure was carried out to provide an estimate of the overall accuracy of the predictions. Blind samples from 10 different French Fries samples prepared in different oxidation degree of oils were used to validate the performance of the proposed method. The TPC, TGP, THP, OTG, and AV of the oils were initially analyzed using AOCS standard method before the proposed FTIR analysis. The results obtained are presented in Fig. 4.

Figure 4 confirms that the two methods agreed with each other well, with $\mathrm{R}^{2}>0.975$ for TPC, TGP, THP, and AV prediction, except for OTG $\left(R^{2}=0.656\right)$. FTIR methods combined with ANN could measure efficiently the TPC, TGP, THP, and AV of various French fries. Therefore, the proposed method can be used as an alternative TPC, TGP, THP, and AV determination method.
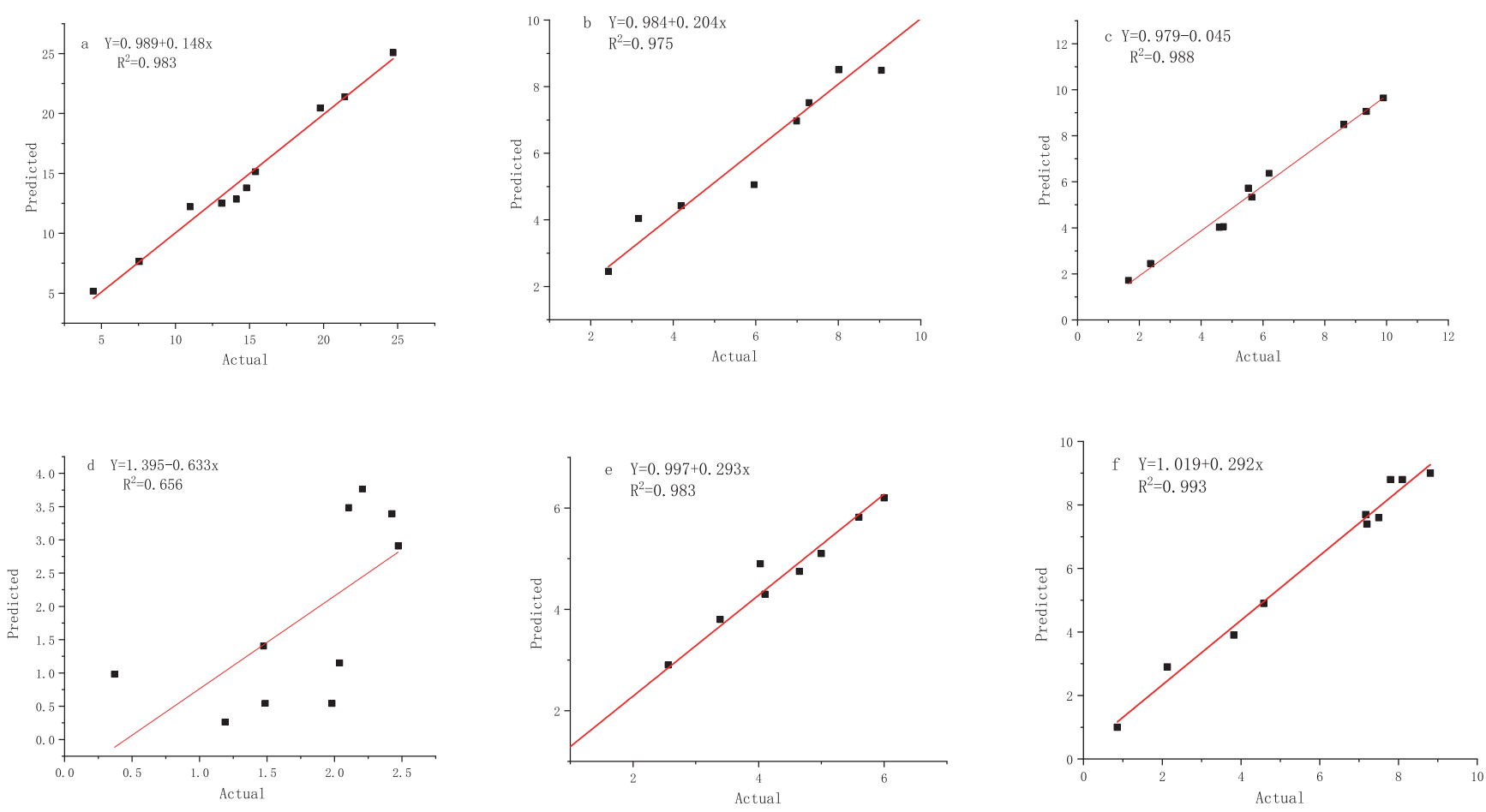

Fig. 4 Establishment of external validation model predicted by BP-ANN and the actual value measured by standard method. (a) TPC; (b)TGP; (c)THP; (d) OTG; (e) AV; (f) PV. 


\section{Conclusions}

This study aimed to simplify oxidation parameters determination in French fries using a rapid and convenient PE film-based FTIR procedure, which are considered practical for routine implementation. Furthermore, compared with PLS model, the BP-ANN model showed good performance and excellent prediction for the changes of quality (TPC, TGP, THP, and AV) in French fries during frying based on FTIR spectroscopy. FTIR spectral regions correspond to $\mathrm{ROOH}, \mathrm{FFA}(\mathrm{C}=\mathrm{O}$ bond $)$, and $-\mathrm{HC}=\mathrm{CH}$-(trans) functional groups were selected for the model establishment. With the increase of the FTIR absorbance intensity in these regions, a deeper degree of thermal oxidative decomposition was presented in French fries during frying. The proposed method indicated that the quality of F-PO was better than F-SuO and F-RO, followed by F-SO. This investigation opens new opportunities for developing FTIR spectroscopy combined with BP-ANN as a promising method for the qualitative detection of fried food in a wide range of applications.

\section{Acknowledgement}

This work was financially supported by the National First-Class Discipline Program of Food Science and Technology (JUFSTR20180202); Postgraduate Research \& Practice Innovation Program of Jiangsu Province (KYCX20_1852); National Natural Science Foundation of China (31901728); Jiangsu Planned Projects for Postdoctoral Research Funds (2020Z297). We thank Jianhua Huang, Ting Shi, and Zhe Dong for assisting in preparation of this manuscript.

\section{Conflict of Interest}

The authors declare that they have no conflict of interest.

\section{Supporting Information}

This material is available free of charge via the Internet at doi: 10.5650/jos.ess21006

\section{References}

1) Sumnu, S.G.; Sahin, S. Advances in deep-fat frying offoods. CRC Press (2008).

2) Erickson, M.D. Deep frying: Deep frying chemistry, nutrition, and practical application, $2^{\text {nd }}$ ed., AOCS Press, Champagne, IL (2007).
3) Gupta, M.K.; Warner, K.; White, P.J.; Gupta, M.K.; Warner, K.; White, P.J. Frying technology and practices. AOCS press, Urbana, Chapters 2, 12 (2004).

4) Choe, E.; Min, D.B. Chemistry of deep-fat frying oils. J. Food Sci. 72, R77-R86(2007).

5) Tabee, E.; Jägerstad, M.; Dutta, P.C. Frying quality characteristics of French fries prepared in refined olive oil and palm olein. J. Am. Oil Chem. Soc. 86, 885$893(2009)$.

6) Li, X.; Yu, X.; Sun, D.; Li, J.; Wang, Y.; Cao, P.; Liu, Y. Effects of polar compounds generated from the deepfrying process of palm oil on lipid metabolism and glucose tolerance in Kunming mice. J. Agr. Food Chem. 65, 208-215 (2016).

7) Liu, Y.; Wang, Y.; Cao, P.; Liu, Y. Degradation of edible oil during deep-frying process by electron spin resonance spectroscopy and physicochemical appreciation. Eur. J. Lipid Sci. Tech. 120, 1700376 (2018).

8) Yang, D.; Wu, G.; Li, P.; Zhang, H.; Qi, X. Comparative analysis of the oil absorption behavior and microstructural changes of fresh and pre-frozen potato strips during frying via mrl, sem, and xrd. Food Res Int. 122, 295-302 (2019).

9) Li, J.; Cai, W.; Sun, D.; Liu, Y. A quick method for determining total polar compounds of frying oils using electric conductivity. Food Anal. Method. 9, 14441450 (2016).

10) Li, Q.; Chen, J.; Huyan, Z.; Kou, Y.; Xu, L.; Yu, X.; Gao, J.M. Application of Fourier transform infrared spectroscopy for the quality and safety analysis of fats and oils: A review. Crit. Rev. Food Sci. 1-15(2018).

11) Nenadis, N.; Tsimidou, M.Z. Perspective of vibrational spectroscopy analytical methods in on-field/official control of olives and virgin olive oil. Eur. J. Lipid Sci. Tech. 119, 1-18(2017).

12) Sow, L.C.; Kong, K.; Yang, H. Structural modification of fish gelatin by the addition of gellan, $\kappa$-carrageenan, and salts mimics the critical physicochemical properties of pork gelatin. J. Food Sci. 83, 1280-1291 (2018).

13) Sow, L.C.; Chong, J.M.N.; Liao, Q.X.; Yang, H. Effects of $\kappa$-carrageenan on the structure and rheological properties of fish gelatin. J. Food Eng. 239, 92-103 (2018).

14) Chen, J.Y.; Zhang, H.; Ma, J.K.; Tuchiya, T.; Miao, Y.L. Determination of the degree of degradation of frying rapeseed oil using Fourier-transform infrared spectroscopy combined with partial least-squares regression. Int. J. Anal. Chem. 1-6(2015).

15) Talpur, M.Y.; Hassan, S.S.; Sherazi, S.T.H.; Mahesar, S.A.; Kara, H.; Kandhro, A.A. A simplified FTIR chemometric method for simultaneous determination of four oxidation parameters of frying canola oil. Spectrochim Acta A 149, 656-661 (2015).

16) Chen, J.; Zhang, L.; Geng, Q.; Jing, B.; Yu, X. Determi- 
nation of total polar compounds in frying oils by PEFilm-Based FTIR and ATR-FTIR spectroscopy. Eur. J. Lipid Sci. Tech. 120, 1800250 (2018).

17) Yu, P.; Low, M.Y.; Zhou, W. Design of experiments and regression modelling in food flavour and sensory analysis: A review. Trends Food Sci Tech. 71, 202-215 (2018).

18) Sun, Q.; Zhang, M.; Mujumdar, A.S.; Yang, P. Combined LF-NMR and artificial intelligence for continuous realtime monitoring of carrot in microwave vacuum drying. Food Bio. Tech. 1-12 (2019).

19) Goyal, S. Artificial neural networks (ANNs) in food science-A review. Int. J. Scientific World 1(2), 19-28 (2013).

20) Esteki, M.; Simal-Gandara, J.; Shahsavari, Z.; Zandbaaf, S.; Dashtaki, E.; Vander Heyden, Y. A review on the application of chromatographic methods, coupled to chemometrics, for food authentication. Food Control. 93, 165-182 (2018).

21) Ordukaya, E.; Karlik, B. Fruit juice-alcohol mixture analysis using machine learning and electronic nose. IEEJ Trans Electr. Electr. Eng. 11(S1), S171-S176 (2016).

22) Winiczenko, R.; Górnicki, K.; Kaleta, A.; Martynenko, A.; Janaszek-Mańkowska, M.; Trajer, J. Multi-objective optimization of convective drying of apple cubes. Comput. Electron. Agr. 145, 341-348(2018).

23) Karaman, S.; Ozturk, I.; Yalcin, H.; Kayacier, A.; Sagdic, O. Comparison of adaptive neuro-fuzzy inference system and artificial neural networks for estimation of oxidation parameters of sunflower oil added with some natural byproduct extracts. J. Sci. Food Agr. 92, 49-58 (2012).

24) Li, X.; Wu, X.; Liu, R.; Jin, Q.; Wang, X. Effect of frying conditions on fatty acid profile and total polar materials via viscosity. J. Food Eng. 166, 349-355 (2015).

25) American Oil Chemists' Society. AOCS official method Cd 3a-63 acid value method. Official methods and recommended practices of the AOCS. Champaign, IL (2003).

26) American Oil Chemists' Society. AOCS official method Cd 20-91. Official methods and recommended practices of the AOCS. Champaign, IL (1993).
27) Xu, L.; Wu, G.; Zhang, Y.; Wang, Q.; Zhao, C.; Zhang, H.; Jin, Q.; Wang, X. Evaluation of glycerol core aldehydes formation in edible oils under restaurant deep frying. Food Res. Int. 137, 109696 (2020).

28) Dong, X.; Li, Q.; Sun, D.; Chen, X.; Yu, X. Direct FTIR analysis of free fatty acids in edible oils using disposable polyethylene films. Food Anal. Method. 8, 857863(2015).

29) Sun, Q.; Zhang, M.; Mujumdar, A.S. Recent developments of artificial intelligence in drying of fresh food: A review. Crit. Rev. Food Sci. Nutr. 59, 2258-2275 (2019).

30) Xu, L.; Yu, X.; Liu, L.; Li, M.; Zhang, R. A rapid method for evaluating the edible oil oxidative stability during ambient storage by FTIR spectroscopy using a mesh cell. Anal. Methods 8, 5117-5122(2016).

31) Guillén, M.D.; Cabo, N. Some of the most significant changes in the Fourier Transform Infrared Spectra of edible oils under oxidative conditions. J. Sci. Food Agri. 80, 2028-2036 (2000).

32) Sow, L.C.; Tan, S.J.; Yang, H. Rheological properties and structure modification in liquid and gel of tilapia skin gelatin by the addition of low acyl gellan. Food Hydrocoll. 90, 9-18(2019).

33) Sow, L.C.; Yu Toh, N.Z.; Wong, C.W.; Yang, H. Combination of sodium alginate with tilapia fish gelatin for improved texture properties and nanostructure modification. Food Hydrocoll. 94, 459-467 (2019).

34) Cavanna, D.; Righetti, L.; Elliott, C.; Suman, M. The scientific challenges in moving from targeted to nontargeted mass spectrometric methods for food fraud analysis: A proposed validation workflow to bring about a harmonized approach. Trends Food Sci. Tech. 80, 223-241 (2018).

CC BY-SA 4.0 (Attribution-ShareAlike 4.0 International). This license allows users to share and adapt an article, even commercially, as long as appropriate credit is given and the distribution of derivative works is under the same license as the original. That is, this license lets others copy, distribute, modify and reproduce the Article, provided the original source and Authors are credited under the same license as the original. 